PROCEEDINGS OF THE

AMERICAN MATHEMATICAL SOCIETY

Volume 128, Number 8, Pages 2393-2403

S 0002-9939(99)05302-2

Article electronically published on November 29, 1999

\title{
ON THE ENTROPY OF THE CONVEX HULL OF FINITE SETS
}

\author{
IOANNA KYREZI
}

(Communicated by Dale Alspach)

\begin{abstract}
We give estimates for the entropy numbers and the Gel'fand diameters of the symmetric convex hull of a finite number of points in a Banach or a Hilbert space.
\end{abstract}

\section{INTRODUCTION}

Let $(X,\|\cdot\|)$ be a Banach space and let $A$ be a bounded subset of $X$. The covering numbers $N(A ; \varepsilon), \varepsilon>0$, of $A$ are defined by

$$
N(A ; \varepsilon):=\inf \left\{N: \exists x_{1}, \ldots, x_{N} \in M \text { such that } A \subset \bigcup_{k=1}^{N} B\left(x_{k} ; \varepsilon\right)\right\},
$$

the entropy numbers $\varepsilon_{n}(A)$ by

$$
\varepsilon_{n}(A):=\inf \{\varepsilon>0: N(A ; \varepsilon) \leq n\}
$$

and the dyadic entropy numbers $e_{n}(A)$ by

$$
e_{n}(A):=\varepsilon_{2^{n-1}}(A), \quad n=1,2, \ldots .
$$

The dyadic entropy numbers of a bounded linear operator $u: X \rightarrow Y$ between two Banach spaces $X$ and $Y$ are defined by

$$
e_{n}(u):=e_{n}\left(u\left(B_{X}\right)\right), \quad n \in \mathbb{N},
$$

where $B_{X}$ is the unit ball of $X$.

We say that a Banach space $X$ is of type $p, 1<p \leq 2$, if there exists a constant $C>0$, such that for every finite family of points $\left\{x_{1}, x_{2}, \ldots, x_{n}\right\}$ of $X$, we have

$$
\int_{0}^{1}\left\|\sum_{i=1}^{n} r_{i}(t) x_{i}\right\| d t \leq C\left(\sum_{i=1}^{n}\left\|x_{i}\right\|^{p}\right)^{1 / p},
$$

where $\left(r_{i}\right)_{i=1}^{\infty}$ are the Rademacher functions. The smallest constant $C$ which satisfies the previous inequality is the type $p$ constant of $X$, which we denote $\tau_{p}(X)$.

The following theorem is due to B. Maurey (see [P2] and [C]).

Received by the editors June 14, 1998 and, in revised form, September 22, 1998.

2000 Mathematics Subject Classification. Primary 46B07, 46B20, 47B37, 52A38.

Key words and phrases. Metric entropy, entropy numbers, Gel'fand numbers.

The author's research was supported by Hellenic S.S.F. 
Theorem 0.1. Let $X$ be a Banach space of type $p, 1<p \leq 2$, and let us consider an operator $S: \ell_{1}^{N} \rightarrow X$. Then there exists a universal constant $C \geq 1$, i.e. $C$ is independent of $N, S$ and $X$, such that

$$
e_{k}(S) \leq C \tau_{p}(X)\left(\frac{\log \left(\frac{N}{k}+1\right)}{k}\right)^{1-\frac{1}{p}}\|S\|, \quad 1 \leq k \leq N .
$$

The Gel'fand numbers $c_{n}(u)$ of a bounded operator $u: X \rightarrow Y$ between the Banach spaces $X$ and $Y$ are defined by

$$
c_{n}(u):=\inf \left\{\left\|\left.u\right|_{E}\right\|: E \subset X, \operatorname{codim}(E)<n\right\} .
$$

Let $H$ be a Hilbert space and let us consider an operator $S: \ell_{1}^{N} \rightarrow H$. The following estimate is the analogue of (11) for the Gel'fand numbers $c_{k}(S)$ and it is due to Carl and Pajor (see $[\mathrm{CP}]$ :

$$
c_{k}(S) \leq C \sqrt{\frac{\log \left(\frac{N}{k}+1\right)}{k}}\|S\|, \quad 1 \leq k \leq N .
$$

If $S$ is a (closed) subspace $S \subseteq X$, then we denote by $Q_{S}$ the quotient mapping from $X$ onto $X / S$. The Kolmogorov numbers $d_{n}(u)$ of a bounded operator $u: X \rightarrow Y$ between the Banach spaces $X$ and $Y$ are defined by

$$
d_{n}(u):=\inf \left\{\left\|Q_{S} u\right\|: S \subset X, \operatorname{dim}(S)<n\right\} .
$$

We have $c_{k}(u)=d_{k}\left(u^{*}\right)$.

Given a set $A$, we can write down analogues of (1) and (2) which describe the convex hull of $A$. More precisely, given a set $A$, we can consider the Banach space $\ell_{1}(A)$ of all summable families of real numbers, $\left(\xi_{t}\right)_{t \in A}$, indexed over $A$, i.e.

$$
l_{1}(A)=\left\{\left(\xi_{t}\right)_{t \in A}:\left\|\left(\xi_{t}\right)_{t \in A}\right\|=\sum_{t \in A}\left|\xi_{t}\right|<+\infty\right\} .
$$

We denote by $\left(e_{t}\right)_{t \in A}$ the canonical basis of $\ell_{1}(A)$.

Now let us assume that $A$ is a bounded subset of a Banach space $X$ and consider the operator $u: \ell_{1}(A) \rightarrow X$ defined by $u\left(e_{t}\right)=t$. If $B_{\ell_{1}(A)}$ is the unit ball of $\ell_{1}(A)$ and $\operatorname{co}(A)$ is the symmetric convex hull of $A$, then $u\left(B_{\ell_{1}(A)}\right)=\operatorname{co}(A)$ and hence

$$
e_{n}(\operatorname{co}(A))=e_{n}(u) \quad \text { and } \quad c_{n}(\operatorname{co}(A))=c_{n}(u)=d_{n}\left(u^{*}\right) .
$$

Suppose now that $A$ is a subset of a Hilbert space $H$ and that $\operatorname{card}(A)=N$. Then the estimates (11) and (21) imply that

$$
\begin{array}{ll}
e_{k}(\operatorname{co}(A)) \leq C \sqrt{\frac{\log \left(\frac{N}{k}+1\right)}{k}} \operatorname{diam}(A), & 1 \leq k \leq N, \\
c_{k}(\operatorname{co}(A)) \leq C \sqrt{\frac{\log \left(\frac{N}{k}+1\right)}{k}} \operatorname{diam}(A), & 1 \leq k \leq N,
\end{array}
$$

where $\operatorname{diam}(A)$ denotes the diameter of $A$.

If $A$ is a subset of a Banach space $X$ of type $p, 1<p \leq 2$, with $\operatorname{card}(A)=N$, then Theorem 0.1 implies that

$$
e_{k}(\operatorname{co}(A)) \leq C \tau_{p}(X)\left(\frac{\log \left(\frac{N}{k}+1\right)}{k}\right)^{1-\frac{1}{p}} \operatorname{diam}(A), \quad 1 \leq k \leq N .
$$


We want to obtain variants of the estimates (3), (4) or (5) which take into consideration not only the diameter of $A$ but also the distribution of the points of $A$ in the space, i.e. the decay of the entropy numbers $\varepsilon_{n}(A)$ of $A$. This is, for example, the case when $A=\operatorname{co}\left(\left\{x_{k}\right\}_{k \geq 1}\right)$ where $\left\{x_{k}\right\}_{k \geq 1} \subseteq X$ is a sequence of points in $X$ such that $\left\|x_{k}\right\| \leq \phi(k), k \geq 1$, where $\phi$ is a positive decreasing function.

In this paper we obtain some results in the case where the entropy numbers $\varepsilon_{n}(A)$ of $A$ do not decrease too fast as $n \rightarrow+\infty$. More precisely, let $\phi$ be a positive decreasing function on $\mathbb{N}^{*}$ which satisfies for some $\alpha>0$,

$$
\phi(k) \leq \alpha \phi(2 k), \quad k \geq 1 .
$$

Let $A \subseteq X$ be a finite subset of a Banach space $X$ with $\operatorname{card}(A)=N$ and let us assume that

$$
\varepsilon_{k}(A) \leq \phi(k), \quad 1 \leq k \leq N
$$

In this article, we give some estimates of the dyadic entropy numbers $e_{k}(\operatorname{co}(A))$ and of the Gel'fand numbers $c_{k}(\operatorname{co}(A))$ in terms of $\phi(k)$. The proofs are inspired by ideas from $[\mathrm{BP}$.

In the first section we treat the case of an arbitrary Banach space $X$ and in the second, the case of a Hilbert space and of a Banach space of type $p, 1<p \leq 2$.

An interesting example of a function $\phi$ which satisfies (6) is given by

$$
\phi(k)=C k^{-\gamma_{1}}(\ln (k+1))^{-\gamma_{2}}, \quad k=1,2, \ldots,
$$

for $\gamma_{1}, \gamma_{2} \geq 0$ or for $\gamma_{1}>0, \gamma_{2} \in \mathbb{R}$.

Throughout this article, $\phi$ will be a positive decreasing function on $\mathbb{N}^{*}$, satisfying the property (6) for a fixed $\alpha>0$. Note that (6) easily implies that

$$
\phi(m) \leq \alpha^{\left[\log _{2} \frac{k}{m}\right]+1} \phi(k),
$$

for any $m \leq k$.

\section{Case of an arbitrary space}

Theorem 1.1. Let $A$ be a finite subset of the unit ball of a Banach space $X$ with $\operatorname{card}(A)=N$ and such that

$$
\varepsilon_{k}(A) \leq \phi(k), \quad k=1,2, \ldots, N .
$$

Then there exists $C(\alpha)>0$ independent of $N$, such that

$$
c_{k}(\operatorname{co}(A)) \leq C(\alpha) \phi(k), \quad 1 \leq k \leq N .
$$

Proof. Since $A$ is contained in the unit ball of $X$, we have

$$
c_{k}(u) \leq c_{1}(u) \leq 1 .
$$

So, if we set $C_{1}=1 / \phi(6)$, the decay of the function $\phi$ implies that

$$
c_{k}(\operatorname{co}(A)) \leq 1 \leq C_{1} \phi(6) \leq C_{1} \phi(k),
$$

for all $k \leq \min \{6, N\}$.

If $6<k \leq N$, then we set

$$
m=\left[\frac{k}{3}\right] \quad \text { and } \quad r=\left[\frac{k}{2}\right] .
$$


Note that $m \leq k / 2$. Let $\Gamma_{1} \subseteq A$ be a $2 \varepsilon_{m}(A)$-net of $A$ such that $\operatorname{card}\left(\Gamma_{1}\right) \leq m$. For every $t \in A$, we denote by $\zeta(t)$ an element of $\Gamma_{1}$ such that $\|\zeta(t)-t\| \leq 2 \varepsilon_{m}(A)$ and we define

$$
\Gamma_{2}=\{t-\zeta(t): t \in A\} .
$$

Then $\operatorname{co}(A) \subseteq \operatorname{co}\left(\Gamma_{1}\right)+\operatorname{co}\left(\Gamma_{2}\right)$ and hence, using a classical property of Gel'fand numbers,

$$
c_{k}(\operatorname{co}(A)) \leq c_{r+1}\left(\operatorname{co}\left(\Gamma_{1}\right)\right)+c_{r}\left(\operatorname{co}\left(\Gamma_{2}\right)\right) .
$$

Since $r+1>m$ and $\operatorname{card}\left(\Gamma_{1}\right) \leq m$, we have

$$
c_{r+1}\left(\operatorname{co}\left(\Gamma_{1}\right)\right)=0 .
$$

To estimate the remaining term $c_{r}\left(\operatorname{co}\left(\Gamma_{2}\right)\right)$, we use the inequality (7) to get

$$
c_{r}\left(\operatorname{co}\left(\Gamma_{2}\right)\right) \leq \sup _{s \in \Gamma_{2}}\|s\| \leq 2 \varepsilon_{m}(A) \leq 2 \phi(m) \leq 2 \alpha^{\left[\log _{2} \frac{k}{m}\right]+1} \phi(k) .
$$

Since $k>6$, we can see that $\frac{k}{m} \leq 6$, and hence

$$
c_{r}\left(\operatorname{co}\left(\Gamma_{2}\right)\right) \leq 2 \alpha^{\left[\log _{2} 6\right]+1} \phi(k) .
$$

Theorem 1.1 follows from (9), (10) and (11) by taking

$$
C(\alpha)=\max \left\{C_{1}, 2 \alpha^{\left[\log _{2} 6\right]+1}\right\} .
$$

The following result is a variant of Carl's inequality (for a proof see CKP Theorem 1.3]).

Proposition 1.2. Let $\left(b_{n}\right)$ be a positive and nondecreasing sequence with the property that there is a constant $\gamma \geq 1$ such that $b_{2 n} \leq \gamma b_{n}$ for all $n \in \mathbb{N}$. Then there exists a constant $c(\gamma) \geq 1$ such that for every bounded $u: X \rightarrow Y$ between the Banach spaces $X$ and $Y$ and all $n \in \mathbb{N}$,

$$
\sup _{1 \leq k \leq n} b_{k} e_{k}(u) \leq c(\gamma) \sup _{1 \leq k \leq n} b_{k} s_{k}(u),
$$

where $s_{k}$ denotes either $c_{k}$ or $d_{k}$.

Using Proposition 1.2 and Theorem 1.1, we can obtain the following estimate on the entropy numbers of the symmetric convex hull of $A$ and its dual version.

Theorem 1.3. Let $A$ be a finite subset of the unit ball of a Banach space $X$ with $\operatorname{card}(A)=N$ and such that

$$
\varepsilon_{k}(A) \leq \phi(k), \quad k=1,2, \ldots, N .
$$

(i) There exists $C(\alpha)>0$ independent of $N$, such that

$$
e_{k}(\operatorname{co}(A)) \leq C(\alpha) \phi(k), \quad 1 \leq k \leq N .
$$

(ii) If $u: \ell_{1}(A) \rightarrow X$ is the bounded operator defined by $u\left(e_{t}\right)=t, t \in A$, there exists $C(\alpha)>0$ independent of $N$, such that

$$
e_{k}\left(u^{*}\right) \leq C_{\alpha} \phi(k), \quad 1 \leq k \leq N .
$$


Proof of (i). Theorem 1.1 implies that for every integer $n \in \mathbb{N}$,

$$
\sup _{k \leq N} \frac{1}{\phi(k)} c_{k}(\operatorname{co}(A)) \leq C(\alpha) .
$$

Now we observe that the sequence $b_{k}=1 / \phi(k)$ is a positive, nondecreasing sequence satisfying $b_{2 k} \leq \gamma b_{k}, k \in \mathbb{N}$, with $\gamma=\alpha$.

So, by Proposition [1.2, there exists a function $C^{\prime}(\alpha)>0$ such that

$$
\sup _{k \leq N} \frac{1}{\phi(k)} e_{k}(\operatorname{co}(A)) \leq C^{\prime}(\alpha) \sup _{k \leq N} \frac{1}{\phi(k)} c_{k}(\operatorname{co}(A)) \leq C^{\prime}(\alpha) C(\alpha)
$$

and hence for all $1 \leq k \leq N$

$$
e_{k}(\operatorname{co}(A)) \leq C^{\prime}(\alpha) C(\alpha) \phi(k)
$$

and the first assertion follows.

Proof of (ii). Since $c_{k}(u)=d_{k}\left(u^{*}\right)$, the proof of (ii) follows exactly the same lines.

Remark. Since the conclusions of Theorems 1.1 and 1.3 do not depend on the cardinal $N$ of $A$, we can extend these results to the case of arbitrary subsets of $X$. Thus, we can obtain an elementary proof of a result in CKP.

The following result is an immediate application of Theorems 1.1 and 1.3

Corollary 1.4. Let $A$ be a subset of the unit ball of a Banach space $X$ such that

$$
\varepsilon_{k}(A) \leq C k^{-\gamma_{1}}(\ln (k+1))^{-\gamma_{2}}, \quad k=1,2, \ldots,
$$

for $\gamma_{1}, \gamma_{2} \geq 0$ or for $\gamma_{1}>0, \gamma_{2} \in \mathbb{R}$. Then, there exists a constant $C^{\prime}=C\left(\gamma_{1}, \gamma_{2}\right)>$ 0 such that

$$
\max \left\{e_{k}(\operatorname{co}(A)), c_{k}(\operatorname{co}(A))\right\} \leq C^{\prime} k^{-\gamma_{1}}(\ln (k+1))^{-\gamma_{2}}, \quad k \geq 1 .
$$

\section{Case of Hilbert spaces}

Theorem 2.1. Let $A$ be a finite subset of the unit ball of a Hilbert space $H$ with $\operatorname{card}(A)=N$ and such that

$$
\varepsilon_{k}(A) \leq \phi(k), \quad k=1,2, \ldots, N .
$$

Then there exists $C(\alpha)>0$ independent of $N$, such that

$$
c_{k}(\operatorname{co}(A)) \leq C(\alpha) \sqrt{\frac{\ln \left(\frac{N}{k}+1\right)}{k}} \phi(k), \quad 1 \leq k \leq N .
$$

Proof. First we observe that, since $A$ is contained in the unit ball of $H$, we have

$$
c_{k}(\operatorname{co}(A)) \leq c_{1}(\operatorname{co}(A)) \leq 1
$$

So, if we set $C_{1}=\sqrt{6} /(\phi(6) \sqrt{\ln 2})$, then the decay of the function $\phi$ gives

$$
c_{k}(\operatorname{co}(A)) \leq 1 \leq C_{1} \sqrt{\frac{\ln \left(\frac{N}{k}+1\right)}{k}} \phi(6) \leq C_{1} \sqrt{\frac{\ln \left(\frac{N}{k}+1\right)}{k}} \phi(k),
$$

for all $k \leq \min \{6, N\}$.

If $6<k \leq N$, then we proceed as in the proof of Theorem 1.1 and we set

$$
m=\left[\frac{k}{3}\right] \leq \frac{k}{2}, \quad r=\left[\frac{k}{2}\right]
$$


Let us consider $\Gamma_{1} \subseteq A$, a $2 \varepsilon_{m}(A)$-net of $A$, such that $\operatorname{card}\left(\Gamma_{1}\right) \leq m$. For any $t \in A$, we denote by $\zeta(t)$ an element of $\Gamma_{1}$ such that $\|\zeta(t)-t\| \leq 2 \varepsilon_{m}(A)$ and we define the set

$$
\Gamma_{2}=\{t-\zeta(t): t \in A\} .
$$

Then $\operatorname{co}(A) \subseteq \operatorname{co}\left(\Gamma_{1}\right)+\operatorname{co}\left(\Gamma_{2}\right)$, which implies that

$$
c_{k}(\operatorname{co}(A)) \leq c_{r+1}\left(\operatorname{co}\left(\Gamma_{1}\right)\right)+c_{r}\left(\operatorname{co}\left(\Gamma_{2}\right)\right) .
$$

Since $r+1>m$ and $\operatorname{card}\left(\Gamma_{1}\right) \leq m$, we obtain

$$
c_{r+1}\left(\operatorname{co}\left(\Gamma_{1}\right)\right)=0 .
$$

To estimate $c_{r}\left(\operatorname{co}\left(\Gamma_{2}\right)\right)$, we use Theorem 0.1 and the property (7) of $\phi$, to obtain

$$
\begin{aligned}
c_{r}\left(\operatorname{co}\left(\Gamma_{2}\right)\right) & \leq C_{m} \sqrt{\frac{\ln \left(\frac{N}{r}+1\right)}{r}} \sup _{s \in \Gamma_{2}}\|s\| \leq C_{M} \sqrt{\frac{\ln \left(\frac{N}{r}+1\right)}{r}} 2 \varepsilon_{m}(A) \\
& \leq C_{M} \sqrt{\frac{\ln \left(\frac{N}{r}+1\right)}{r}} 2 \phi(m) \leq C_{M} \sqrt{\frac{\ln \left(\frac{N}{r}+1\right)}{r}} 2 \alpha^{\left[\log _{2} \frac{k}{m}\right]+1} \phi(k),
\end{aligned}
$$

where $C_{M}$ is the numerical constant (independent of $N$ and of $k$ ) of Theorem 0.1 Now since $k>6$, we have $\left[\frac{k}{2}\right] \geq \frac{k}{3}$ and $\frac{k}{m} \leq 6$ and hence

$$
\begin{aligned}
c_{r}\left(\operatorname{co}\left(\Gamma_{2}\right)\right) & \leq C_{M} \sqrt{3} \sqrt{\frac{\ln \left(3 \frac{N}{k}+1\right)}{k}} 2 \alpha^{\left[\log _{2} 6\right]+1} \phi(k) \\
& \leq C_{M} 6 \alpha^{\left[\log _{2} 6\right]+1} \sqrt{\frac{\ln \left(\frac{N}{k}+1\right)}{k}} \phi(k) .
\end{aligned}
$$

The estimates (16), (17) and (18) imply the assertion of the theorem with the constant

$$
C(\alpha)=\max \left\{C_{1}, 6 C_{M} \alpha^{\left.\log _{2} 6\right]+1}\right\}
$$

The following result gives the corresponding estimate for the entropy numbers of the convex symmetric hull of $A \subseteq H$.

Theorem 2.2. Let $A$ be a finite subset of the unit ball of a Hilbert space $H$ such that $\operatorname{card}(A)=N$ and such that

$$
\varepsilon_{k}(A) \leq \phi(k), \quad k=1,2, \ldots, N .
$$

(i) There exists $C(\alpha)>0$ independent of $N$, such that

$$
e_{k}(\operatorname{co}(A)) \leq C(\alpha) \sqrt{\frac{\ln \left(\frac{N}{k}+1\right)}{k}} \phi(k), \quad 1 \leq k \leq N .
$$

(ii) If $u: \ell_{1}(A) \rightarrow H$ is the bounded operator defined by $u\left(e_{t}\right)=t, t \in A$, there exists $C(\alpha)>0$ independent of $N$, such that

$$
e_{k}\left(u^{*}\right) \leq C(\alpha) \sqrt{\frac{\ln \left(\frac{N}{k}+1\right)}{k}} \phi(k), \quad 1 \leq k \leq N .
$$

When $k>N$ a better estimate than (19) is given by the following result ([Pie], P1], [CS] or $[\mathrm{BP}]$ ). 
Proposition 2.3. If $T: X \rightarrow Y$ is an operator of rank $n$ and $k \geq n$, then

$$
e_{k}(T) \leq 8 \cdot 2^{-\frac{k}{n}} e_{n}(T) .
$$

Proof of Theorem 2.2 (i). We shall prove by induction on the cardinal $N$ of $A$ that there exists $C(\alpha)>0$ such that the following statement, denoted by $\mathbb{P}(N)$, is true:

$$
\begin{gathered}
\forall A \subseteq X \text { such that } \operatorname{card}(A)=N \text { and such that } \\
\qquad \forall \leq \leq N, \varepsilon_{k}(A) \leq \phi(k), \\
\text { then } \forall k \leq N, e_{k}(\operatorname{co}(A)) \leq C(\alpha) \sqrt{\frac{\ln \left(\frac{N}{k}+1\right)}{k}} \phi(k) .
\end{gathered}
$$

Fix a constant $\beta \geq 3$, to be determined later, and set $C_{\beta}=\sqrt{2 \beta} /(\phi(2 \beta) \sqrt{\ln 2})$. Since $A$ is contained in the unit ball of $H$ and since the function $\phi$ is decreasing, we have

$$
\begin{aligned}
e_{k}(\operatorname{co}(A)) & \leq e_{1}(\operatorname{co}(A)) \leq 1 \leq C_{\beta} \sqrt{\frac{\ln \left(\frac{N}{k}+1\right)}{k}} \phi(2 \beta) \\
& \leq C_{\beta} \sqrt{\frac{\ln \left(\frac{N}{k}+1\right)}{k}} \phi(k),
\end{aligned}
$$

for all $k \leq 2 \beta$. So, for all $N \leq 2 \beta, \mathbb{P}(N)$ holds for $C(\alpha) \geq C_{\beta}$.

Now let $N_{0}>2 \beta$ and suppose that $\mathbb{P}(N)$ is true for all $N<N_{0}$. We shall prove that $\mathbb{P}\left(N_{0}\right)$ is also true.

If $k \leq 2 \beta$, then by (21),

$$
e_{k}(\operatorname{co}(A)) \leq C_{\beta} \sqrt{\frac{\ln \left(\frac{N}{k}+1\right)}{k}} \phi(k) .
$$

If $2 \beta<k \leq N_{0}$, then we proceed again as in the proof of Theorem 1.3 and we set

$$
m=\left[\frac{k}{\beta}\right] \leq \frac{k}{2}, \quad r=\left[\frac{k}{2}\right] .
$$

Next we consider $\Gamma_{1} \subseteq A$, a $2 \varepsilon_{m}$-net of $A$, such that $\operatorname{card}\left(\Gamma_{1}\right)=m$. For any $t \in A$, we choose an element $\zeta(t)$ of $\Gamma_{1}$ such that $\|\zeta(t)-t\| \leq 2 \varepsilon_{m}$ and we define the set

$$
\Gamma_{2}=\{t-\zeta(t): t \in A\} .
$$

Then $\operatorname{co}(A) \subseteq \operatorname{co}\left(\Gamma_{1}\right)+\operatorname{co}\left(\Gamma_{2}\right)$, which implies

$$
e_{k}(\operatorname{co}(A)) \leq e_{r+1}\left(\operatorname{co}\left(\Gamma_{1}\right)\right)+e_{r}\left(\operatorname{co}\left(\Gamma_{2}\right)\right) .
$$

To estimate $e_{r}\left(\operatorname{co}\left(\Gamma_{2}\right)\right)$, we use Theorem 0.1 and the inequality (7), to obtain

$$
\begin{aligned}
e_{r}\left(\operatorname{co}\left(\Gamma_{2}\right)\right) & \leq C_{M} \sqrt{\frac{\ln \left(\frac{N}{r}+1\right)}{r}} \sup _{s \in \Gamma_{2}}\|s\| \leq C_{M} \sqrt{\frac{\ln \left(\frac{N}{r}+1\right)}{r}} 2 \varepsilon_{m}(A) \\
& \leq C_{M} \sqrt{\frac{\ln \left(\frac{N}{r}+1\right)}{r}} 2 \phi(m) \leq C_{M} \sqrt{\frac{\ln \left(\frac{N}{r}+1\right)}{r}} 2 \alpha^{\left[\log _{2} \frac{k}{m}\right]+1} \phi(k),
\end{aligned}
$$


where $C_{M}$ is the numerical constant (independent of $N$ and of $k$ ) of Theorem 0.1 Since $k>2 \beta$, we can see that $\left[\frac{k}{2}\right] \geq \frac{k}{3}$ and $\frac{k}{m} \leq 2 \beta$ and hence

$$
e_{r}\left(\operatorname{co}\left(\Gamma_{2}\right)\right) \leq 6 C_{M} \alpha^{\left[\log _{2} 2 \beta\right]+1} \sqrt{\frac{\ln \left(\frac{N}{k}+1\right)}{k}} \phi(k) .
$$

To estimate the remaining term $e_{r+1}\left(\operatorname{co}\left(\Gamma_{1}\right)\right)$, since $\operatorname{card}\left(\Gamma_{1}\right) \leq m<r+1$ and $\frac{r+1}{m} \geq \frac{\beta}{2}$, Proposition 2.3 implies

$$
e_{r+1}\left(\operatorname{co}\left(\Gamma_{1}\right)\right) \leq 8 \cdot 2^{-\frac{r+1}{m}} e_{m}\left(\operatorname{co}\left(\Gamma_{1}\right)\right) \leq 8 \cdot 2^{-\frac{\beta}{2}} e_{m}\left(\operatorname{co}\left(\Gamma_{1}\right)\right) .
$$

Now, $\Gamma_{1} \subseteq A$ and so $\varepsilon_{k}\left(\Gamma_{1}\right) \leq \phi(k)$ for all $k \leq N_{0}$. Moreover, $\operatorname{card}\left(\Gamma_{1}\right)=m$ and $m<N_{0}$. So by the induction hypothesis, $\mathbb{P}(m)$ is true for $\Gamma_{1}$, which implies

$$
e_{m}\left(\operatorname{co}\left(\Gamma_{1}\right)\right) \leq C(\alpha) \sqrt{\frac{\ln 2}{m}} \phi(m) .
$$

Using again the inequality (7), we have

$$
\begin{aligned}
e_{r+1}\left(\operatorname{co}\left(\Gamma_{1}\right)\right) & \leq 8 \cdot 2^{-\frac{\beta}{2}} \sqrt{\frac{\ln 2}{m}} e_{m}\left(\operatorname{co}\left(\Gamma_{1}\right)\right) \leq 8 \sqrt{2} 2^{-\frac{\beta}{2}} C(\alpha) \frac{1}{\sqrt{m}} \phi(m) \\
& \leq 8 \sqrt{2} 2^{-\frac{\beta}{2}} C(\alpha) \alpha^{\left[\log _{2} \frac{k}{m}\right]+1} \frac{1}{\sqrt{m}} \phi(k) .
\end{aligned}
$$

Since $k>2 \beta$, we have $\frac{k}{m} \leq 2 \beta$ and $\left[\frac{k}{\beta}\right] \geq \frac{k}{2 \beta}$ and hence

$$
e_{r+1}\left(\operatorname{co}\left(\Gamma_{1}\right)\right) \leq 8 \sqrt{2} 2^{-\frac{\beta}{2}} \sqrt{2 \beta} \alpha^{\left[\log _{2} 2 \beta\right]+1} C(\alpha) \frac{1}{\sqrt{k}} \phi(k) .
$$

So, we have

$$
\begin{aligned}
e_{k}(\operatorname{co}(A)) & \leq e_{r+1}\left(\operatorname{co}\left(\Gamma_{1}\right)\right)+e_{r}\left(\operatorname{co}\left(\Gamma_{2}\right)\right) \\
& \leq 16 \sqrt{\beta} 2^{-\frac{\beta}{2}} \alpha^{\left[\log _{2} 2 \beta\right]+1} C(\alpha) \frac{1}{\sqrt{k}} \phi(k)+6 C_{M} \alpha^{\left[\log _{2} 2 \beta\right]+1} \sqrt{\frac{\ln \left(\frac{N}{k}+1\right)}{k}} \phi(k) \\
& \leq\left(16 \sqrt{\beta} 2^{-\frac{\beta}{2}} \alpha^{\left[\log _{2} 2 \beta\right]+1} C(\alpha)+6 C_{M} \alpha^{\left[\log _{2} 2 \beta\right]+1}\right) \sqrt{\frac{\ln \left(\frac{N}{k}+1\right)}{k}} \phi(k) .
\end{aligned}
$$

We now choose $\beta$ satisfying

$$
\beta>3 \quad \text { and } \quad 16 \sqrt{\beta} 2^{-\frac{\beta}{2}}\left(\alpha^{\left.\log _{2} 2 \beta\right]+1}\right)<\frac{1}{2} .
$$

We set

$$
C(\alpha)=C_{\beta}+12 C_{M} \alpha^{\left[\log _{2} 2 \beta\right]+1} .
$$

Then we obtain

$$
C>C_{\beta} \text { and } C>16 \sqrt{\beta} 2^{-\frac{\beta}{2}} \alpha^{\left[\log _{2} 2 \beta\right]+1} C(\alpha)+6 C_{M} \alpha^{\left[\log _{2} 2 \beta\right]+1}
$$

and consequently

$$
e_{k}(\operatorname{co}(A)) \leq C(\alpha) \sqrt{\frac{\ln \left(\frac{N}{k}+1\right)}{k}} \phi(k), \quad 1 \leq k \leq N_{0} .
$$

This proves $\mathbb{P}\left(N_{0}\right)$, and (19) follows. 
Remark. We can see that also in this case, the estimates concerning the entropy numbers can be derived from those concerning the Gel'fand numbers, by using Proposition 1.2. We decided to give the above proof because it is more direct.

Proof of Theorem 2.2 (ii). The proof of (20) is similar to the proof of (13). The only difference is that we use the sequence $b_{k}=\sqrt{k} /\left(\sqrt{\log \left(\frac{N}{k}+1\right)} \phi(k)\right)$ instead of the sequence $b_{k}=1 / \phi(k)$. We shall omit the details.

Corollary 2.4. Let $A$ be a finite subset of the unit ball of a Hilbert space $H$ with $\operatorname{card}(A)=N$ and such that

$$
\varepsilon_{k}(A) \leq C k^{-\gamma_{1}}(\ln (k+1))^{-\gamma_{2}}, \quad k=1,2, \ldots, N
$$

for $\gamma_{1}, \gamma_{2} \geq 0$ or for $\gamma_{1}>0, \gamma_{2} \in \mathbb{R}$. Then there exists a constant $C^{\prime}=C\left(\gamma_{1}, \gamma_{2}\right)>0$ such that

$$
\max \left\{e_{k}(\operatorname{co}(A)), c_{k}(\operatorname{co}(A))\right\} \leq C^{\prime} \sqrt{\frac{\ln \left(\frac{N}{k}+1\right)}{k}} k^{-\gamma_{1}}(\ln (k+1))^{-\gamma_{2}}, \quad 1 \leq k \leq N .
$$

The following result is the analogous version to Theorem 2.2 for Banach spaces of type $p, 1<p \leq 2$, and can be proved in the same way.

Theorem 2.5. Let $A$ be a finite subset of the unit ball of a Banach space $X$ of type $p, 1<p \leq 2$, with $\operatorname{card}(A)=N$ and such that

$$
\varepsilon_{k}(A) \leq \phi(k), \quad k=1,2, \ldots, N .
$$

(i) There exists a constant $C=C(p, \alpha)>0$ such that

$$
e_{k}(\operatorname{co}(A)) \leq C\left(\frac{\ln \left(\frac{N}{k}+1\right)}{k}\right)^{1-\frac{1}{p}} \phi(k), \quad 1 \leq k \leq N .
$$

(ii) If $u: \ell_{1}(A) \rightarrow X$ is the bounded operator defined by $u\left(e_{t}\right)=t, t \in A$, there exists a constant $C=C(p, \alpha)>0$ such that

$$
e_{k}\left(u^{*}\right) \leq C\left(\frac{\ln \left(\frac{N}{k}+1\right)}{k}\right)^{1-\frac{1}{p}} \phi(k), \quad 1 \leq k \leq N .
$$

Corollary 2.6. Let $A$ be a finite subset of the unit ball of a Banach space X of type $p, 1 \leq p \leq 2$, with $\operatorname{card}(A)=N$ and such that

$$
\varepsilon_{k}(A) \leq C k^{-\gamma_{1}}(\ln (k+1))^{-\gamma_{2}}, \quad k=1,2, \ldots, N
$$

for $\gamma_{1}, \gamma_{2} \geq 0$ or for $\gamma_{1}>0, \gamma_{2} \in \mathbb{R}$. Then there exists a constant $C^{\prime}=$ $C\left(p, \gamma_{1}, \gamma_{2}\right)>0$ such that

$$
e_{k}(\operatorname{co}(A)) \leq C^{\prime}\left(\frac{\ln \left(\frac{N}{k}+1\right)}{k}\right)^{1-\frac{1}{p}} k^{-\gamma_{1}}(\ln (k+1))^{-\gamma_{2}}, \quad 1 \leq k \leq N .
$$

Remark. We could not prove an analogue of Theorem 2.5(i) for Gel'fand numbers, because there does not exist an estimate similar to the estimate (2) for Banach spaces of type $p, 1<p \leq 2$. Nevertheless, we can prove such a result for the smaller class of Banach spaces $X=\left[X_{1}, H\right]_{\frac{2 p-2}{p}, p}$, i.e. the $\left(\frac{2 p-2}{p}, p\right)$-interpolates of a Banach space $X_{1}$ and a Hilbert space $H$, by using a result on the interpolation of Gel'fand numbers [Pie, Proposition 11.5.8]. 
Let us recall that for $K \subseteq \mathbb{R}^{n}$, the polar set $K^{\circ}$ of $K$ is defined by

$$
K^{\circ}=\left\{x \in \mathbb{R}^{n}:\langle x, y\rangle \leq 1 \forall y \in K\right\} .
$$

The Santaló inequality $[\mathbf{S}]$ and the inverse Santaló inequality $[\mathrm{BM}]$ compare the volume of a compact symmetric convex set $B \subseteq \mathbb{R}^{n}$ with a non-empty interior with the volume of its polar $B^{\circ}$, in the following way:

There are positive constants $D_{1}$ and $D_{2}$ (independent of $n$ and $B$ ) such that

$$
D_{1} / n \leq\left(\operatorname{vol}(B) \operatorname{vol}\left(B^{\circ}\right)\right)^{\frac{1}{n}} \leq D_{2} / n .
$$

Using the above inequalities, the previous results concerning the entropy numbers of $\operatorname{co}(A)$ can provide volumic estimates for $\operatorname{co}(A)$ and its polar $(\operatorname{co}(A))^{\circ}$.

Proposition 2.7. Let $H$ be an n-dimensional Hilbert space and let $A$ be a finite subset of its unit ball with $\operatorname{card}(A)=N \geq n$ and such that

$$
\varepsilon_{k}(A) \leq \phi(k), \quad k=1,2, \ldots, N .
$$

Then there exists a constant $C=C(\alpha)>0$, independent of $n, N$ and $A$, such that

$$
\left(\operatorname{vol}_{n}(\operatorname{co}(A))^{\circ}\right)^{\frac{1}{n}} \geq C\left(\ln \left(\frac{N}{n}+1\right)\right)^{-\frac{1}{2}} \phi(n)^{-1} .
$$

If $A$ is a finite subset of the unit ball of $\ell_{p}^{n}, 1<p \leq 2$, and under the same hypothesis, then there exists $C=C(\alpha, p)>0$ such that

$$
\left(\operatorname{vol}_{n}(\operatorname{co}(A))^{\circ}\right)^{\frac{1}{n}} \geq C\left(\ln \left(\frac{N}{n}+1\right)\right)^{-1+\frac{1}{p}} \phi(n)^{-1} .
$$

Proof. By Theorem 2.2 we have that for any $k \leq N$,

$$
e_{k}(\operatorname{co}(A)) \leq C_{1}(\alpha) \sqrt{\frac{\ln \left(\frac{N}{k}+1\right)}{k}} \phi(k) .
$$

In volumic terms, this means that

$$
\left(\operatorname{vol}_{n}(\operatorname{co}(A))\right)^{\frac{1}{n}} \leq 2 C_{1}(\alpha) \sqrt{\frac{\ln \left(\frac{N}{n}+1\right)}{n}} \phi(n) \frac{C_{2}}{\sqrt{n}} .
$$

Using (26) and (23), we obtain that

$$
\left(\operatorname{vol}_{n}(\operatorname{co}(A))^{\circ}\right)^{\frac{1}{n}} \geq \frac{1}{\left(\operatorname{vol}_{n}(\operatorname{co}(A))\right)^{\frac{1}{n}}} \frac{D_{1}}{n} \geq\left(2 C_{1}(\alpha) C_{2} D_{1}\right) /\left(\sqrt{\ln \left(\frac{N}{n}+1\right)} \phi(n)\right)
$$

which proves (24). The inequality (25) can be proved in the same way.

A result similar to that of Proposition 2.7 has been given in [BP2].

\section{ACKNOWLEDGMENT}

I would like to thank my thesis advisor Professor A. Pajor for his help and encouragement. 


\section{REFERENCES}

[BM] J. Bourgain and V. D. Milman, New volume ratio properties for convex symmetric bodies in $\mathbb{R}^{n}$, Invent. Math. 88 (1987), 319-340. MR 88f:52013

[BP] K. Ball and A. Pajor, The entropy of convex bodies with "few" extreme points, London Math. Soc. Lecture Notes Series 158 (1990), 25-32. MR 93b:46024

[BP2] _ Convex bodies with few faces, Proc. Amer. Math. Soc. 110 (1990), 225-231. MR 90m:52011

[C] B. Carl, Inequalities of Bernstein-Jackson type and the degree of compactness of operators in Banach spaces, Ann. Inst. Fourier 35 (3) (1985), 79-118. MR 86m:47022

[CP] B. Carl and A. Pajor, Gel'fand numbers of operators with values in Hilbert spaces, Invent. Math. 94 (1988), 479-504. MR 90d:46023

[CKP] B. Carl, I. Kyrezi and A. Pajor, Metric entropy of convex hulls in Banach spaces, to appear in the Journal of London Mathematical Society.

[CS] B. Carl and I. Stephani, Entropy, compactness and the approximation of operators, Cambridge University Press (1990). MR 92e:47002

[LT] J. Lindenstrauss and C. Tzafriri, Classical Banach spaces, vol. 1,2, Springer Verlag (1977/79). MR 58:17766 MR 81c:46001

[Pie] A. Pietsch Operator ideals, Berlin (1978), North Holland (1980). MR 81j:47001

[P1] G. Pisier, Volume inequalities in the geometry of Banach spaces, Cambridge University Press (1989).

[P2] Remarques sur un résultat non publié de B. Maurey, Séminaire d'Analyse Fonctionnelle, Ecole Polytechnique-Palaiseau, Exposé 5 (1980/1981). MR 83h:46026

[S] L. Santaló, Un invariante afin para los cuerpos convexos den espacio de $n$ dimensiones, Portugal Math. 8 (1949), 155-161.

Université de Marne-la-Vallée, Equipe d’Analyse et de Mathématiques Appliquées, Cité Descartes, 5 Bd Descartes, Champs sur Marne, 77454 Marne-la-Vallée Cedex 2, FrANCE

E-mail address: kyrezi@math.univ-mlv.fr 